\title{
Turning opposition into support to immigration. The role of social norms
}

\author{
Cristina Cattaneo \\ Daniela Grieco \\ $R F F-C M C C$ \\ University of Milano*
}

Pre-Analysis Plan: April 2020

\begin{abstract}
The number of migrants moving from one country to another is bound to increase, and a considerable fraction of these flows will try to access the European countries. At the same time, public opinion towards immigration is often negative. A supportive public approach has many advantages, as it can facilitate an efficient management of the migration flows and improve the integration of migrants in our societies. Building on a theoretical model, we study a set of experimental treatments aimed at promoting support to immigration through a mechanism based on social norms. Our intervention consists of presenting a favourable social norm prevailing among the members of an "ingroup" (the natives) and test its effectiveness in reducing the social distance between a subject belonging to the ingroup and the "outgroup" (the migrants). Receiving a favourable social norm is expected to deliver a clear sign that peers of the ingroup are not against migrants, ultimately affecting behaviour.
\end{abstract}

Survey experiment platform: Online survey

Survey experiment dates: 23/04/2020

*Cristina Cattaneo (cristina.cattaneo@eiee.org), RFF-CMCC European Institute on Economics and the Environment (EIEE) and Centro Euro-Mediterraneo sui Cambiamenti Climatici, via Bergognone 34, 20144 Milan. Daniela Grieco (daniela.grieco@unimi.it), Department of Law, University of Milano, Italy. 


\section{Introduction}

Large numbers of people in destination countries believe that the amount of immigrants is too high and consequently wish to curtail the quantity of inflows. Public opinion plays a crucial role in shaping policies, including those aimed at improving the integration of migrants. The literature has widely debated the reasons behind strong negative feelings rooted in native's attitudes towards migration, concluding that citizens barely form attitudes about immigration using their personal economic situation (Hainmueller and Hopkins, 2014). Economic self-interest motivations have fared poorly compared to economic sociotropic considerations. Immigration-related attitudes are in fact largely influenced by symbolic concerns that affect the nation as a whole. At the same time, natives display striking misperceptions about key features of migrants (Alesina et al., 2018) and given this vacuum, attitudes are largely shaped by the media rhetoric, which mainly portrays migrants in a negative way (Brader et al., 2008). The majority of the existing studies however, analyze what drives the bitter feeling against migrants and only few consider which factors are able to positively influence attitudes and behaviour in favour of migrants. Given that the framing effect could be asymmetric, with information linked to threat producing sizeable effect on attitude, while favourable information having little effect on it (Brader et al., 2008), an important question remains on how to positively affect attitudes and subsequent behaviour.

The objective of this pre-analysis plan is to present a study design that aims to promote support to migrants through a mechanism based on social norms. We design an intervention that presents different social norms regarding favourable behaviour and attitude towards migrants and test their influence on behavioural outcomes.

The hypothesis is that the information on what other people do (or think) with respect to migrants, influences ones' behaviour. Moreover, through the comparison between the treatments, differing on the social norm conveyed, and a control where no social information is provided, we plan to introduce an exogenous variation in beliefs about what other people do and think with respect to migrants, which ultimately should affect actual behaviour.

\section{Experimental design}

We plan to conduct an incentivized survey and recruit Italian respondents through a commercial firm that will interview a sample of subjects, representative of the Italian population. In the survey experiment, we will randomly allocate respondents to different treatments and one control groups.

Respondents in the treatment groups will receive information on the social norm, namely on what other respondents did before them in terms of donation to a charity 
operating in favour of migrants. Moreover, to understand to what extent the social norm is effective in changing donations per se, or due to its capability to convey information on the ingroup attitude towards the outgroup, we will also design a treatment where we directly provide information on the ingroup (favourable) attitude towards the outgroup.

There are different ways to convey a social norm. Croson et al. (2010) randomly assigned high social information (another donor in a previous pilot study contributing a large amount) versus low social information (another donor contributing a small amount). Then they assess beliefs about the descriptive social norm (participants are asked about their belief of the descriptive norm: "how much do you think an average person would contribute?"). High social information needs to be sufficiently high (the level of the other donor's contribution has to be higher than the 90th percentile), but not too extreme (lower than 99th percentile), otherwise it ceases to influence individual contributions (Shang and Croson, 2009).

Alternative ways to convey social information are providing some statistics on the previous donations (e.g. "half of donations were equal or above 50\%") or on the degree of participation of previous donors (e.g. "a certain percentage of previous respondents decided to donate") (Frey and Meier, 2004). A way to induce in subjects different descriptive norms on attitude is manipulating the consensus about the outgroup by giving information on whether peers consider "Definitely OK/not OK/maybe OK to have negative feelings about the outgroup".

Before presenting the treatments, we will tell respondents that they will receive a bonus on top of the standard payment for completing the survey. This bonus could be kept by the respondent, or donated (all or in part) to an organization randomly assigned to the respondent. To contrast the experimental demand effect we follow Bursztyn et al. (2017) and select two organizations, which could either be pro-migration (working for the rights of immigrants in Italy) or anti-migration (which campaigns to reduce migration in Italy): each subject will be randomly assigned to one of them.

Although we are interested primarily in respondents' donation levels in favour of migrants, observing respondents' donations to the anti-immigrant organisation will also shed light on possible backlash effect of our treatments. After respondents receive one of the treatments (or no information in the case of respondents randomly allocated to the control group), we will ask them to choose the amount of the donation to the organization. We will also ask them to choose to sign a petition in favour of migrants, providing them the web-link to the petition. The order of the two outcome variables will be randomized.

We will implement four treatments plus a control: Positive Donation, Median Donation, Positive Attitude and Negative Donation. The exact wording of the treatments to convey the intended message will be fine-tune through a pilot study.

In the Positive Donation treatment, respondents are made aware of a high donation level, like in Croson et al. (2010). In order to collect information on the distribution of 
donations, we will conduct a pilot survey with Italian respondents. Conveying information on a high donation of previous respondents belonging to the same ingroup is expected to induce an increase in respondents' donations by acting as a positive social norm. In fact, subjects learn that other respondents with a high degree of social similarity (i.e. sharing the same nationality, thus being part of the same ingroup) made a costly choice in favour of migrants and are likely to be influenced by their choice.

In the Median Donation treatment, respondents are made aware of the median (50th percentile) donation level to the pro-migration organization of the participants of the same nationality to a previous identical survey. This treatment has the aim of disentangling the effects of conveying information on previous respondents' donation per se from the effect of reporting that particular donation level (i.e. the highest). Since we will administer the pilot and the survey within a very short period, respondents should not differ ex ante in terms of median donations. Thus, any possible significant difference between this treatment and the control should depend on the fact of providing information on previous respondents' donation per se and not on the specific donation level. On the contrary, any possible significant difference between this treatment and Positive Donation treatment will be simply ascribed to the specific donation level reported (the highest vs. the median).

In the Positive Attitude Treatment, respondents are made aware of a positive attitude towards migrants of participants of the same nationality to a previous identical survey. The objective of this treatment is to isolate the effect of learning about the ingroup (positive) attitude towards migrants. This information will be available for respondents in the Positive Donation treatment too, but derives from the information on the donation level. Therefore, subjects might be influenced by the ingroup attitude or simply motivated by the need to conform to the ingroup donation behaviour. To identify the exact wording of the treatment, we will ask in the pilot study questions like: "In your opinion, should the number of immigrants in this country be reduced a lot, reduced a little, kept at current levels, increased a little; increased a lot". Another possible question would be: "In your opinion, what is the impact of immigrants on Italy's welfare? They generate large positive effects; they generate positive effects; they have no impacts; they generate negative effects; they generate large negative effects".

Control treatment. In the Control treatment, respondents will make their donation to the pro-migration organization, as in the previous treatments, but without receiving any information on previous respondents' behaviour or attitude.

We will finally present the Negative Donation treatment, where respondents are made aware of the top donation level to the organization pro-migration but will then be asked to make their donation to the anti-migration organization. The inclusion of this treatment will help us to contrast the experimental demand effect (respondents might behave in favour of migrants just because they anticipate that the survey is built by 
researchers trying to induce behaviours in favour of migrants), but might also provide evidence of backlash effects: people could react to peers' behaviour in favour of migrants by supporting a charity against migrants.

\section{A simple model for donation}

The way we model an individual's decision to donate to (the members of) an outgroup reflects the assumption that individuals have social preferences. This means that they are concerned not only with their own materialistic payoff, but also with the payoffs of others. Each subject donates an amount $x \geq 0$ out of her endowment $e$ and maximizes the following utility function $\mathrm{U}$ :

$$
U=u(e-x)+a v(x, d)-\frac{\beta}{2}(N-x)^{2}
$$

The material utility of private consumption, $u$, is represented by the endowment $e$ minus the donation to the outgroup $x$ and satisfies standard properties: $u$ ' (.) $>0$ and $u$ " $($. 0 . Donating $x$ to the outgroup determines an additional source of utility, $v$, that captures the warm glow of giving (Andreoni, 1990). The utility $v$ is increasing in the contribution $x$ but has decreasing returns: $v_{x}^{\prime}(.,)>0,. v_{x, x}^{\prime \prime}(.,)<$.0 .

The utility $v$ also depends on $d$, that reflects the perceived distance between the ingroup the subject belongs to and the outgroup. We assume that the utility of giving decreases with the distance: $v_{d}^{\prime}(.,)<$.0 . Furthermore $\lim _{d \rightarrow \infty} v(., d)=0$, i.e. there is no warm glow of giving if the distance $d$ is perceived as very high. We assume $d$ to be determined by two elements: the first is the subject's own attitude towards the outgroup, and the second is her belief on ingroup peers' attitude towards the outgroup. This latter can be affected by the information on peers' behaviour and/or attitude towards the outgroup. The parameter $\alpha \geq 0$ denotes the subject's trade-off between pursuing own material interest and enjoying the benefits of helping the outgroup, i.e. the intensity of warm glow.

The third term in the utility function is the loss the subject experiences if her donation departs from the social norm $N$, i.e. the amount donated by ingroup peers. The parameter $\beta$ reflects the steepness of the tradeoff between material incentives and the desire to conform to the ingroup behaviour. The higher is $\beta$, the more the subject cares about following the norm. Note that a strictly positive donation may occur also in case of a subject perceiving a high distance to the outgroup: this happens when subjects derive large utility from conforming to the norm. 


\section{Data and Outcome variables}

The outcomes variables of the experiment are actual behaviours. We will measure incentivized behaviour by collecting real donation to a non-profit organization operating pro-migrants or, in the case of the Negative Donation treatment, to a non-profit organization operating anti-migrants. In particular, we will ask respondents if they wish to donate part of their fixed endowment to a non-profit organization, which works and campaigns for the rights of immigrants in Italy, or alternatively to a non-profit organization which is against the enlargement of the number of immigrants in Italy. The missions of the two organizations will be made salient, and people will be informed that they will be randomly allocated to donate to one of the two organizations.

The second measure of actual behaviour is the respondents' willingness to sign a petition in favour of migrants and their actual signature, providing a link to a real petition. The order of the two outcomes (petition and donation) will be randomized.

After collecting the amount of the personal donation, we will elicit respondents' beliefs on the average donation level of other natives in the same wave. We will also collect information on respondents' beliefs on peers' attitude towards migrants. Attitudes of peers are measured both pre and post treatments and we will present subjects with slightly different formulation of these questions before and after providing the treatments. We will use different questions for measuring peers' attitudes because there is not a unique way to measure attitudes towards migration, being attitudes not specifically related to one single aspect of immigration.

The survey will also allow us to collect information on respondents' socio-demographic characteristics, such as gender, age, political orientation, own attitude towards migrants, opinion on the relevance on major issues, self-perceived position in the social ladder, selfperceived degree of altruism and well-being from helping others, importance of reputation, marital status, number of children, religion, place of residence, monthly income, highest level of education achieved, sector of occupation, employment status, vote in the past elections, own and parents' ethnic origin, degree of interaction ("close acquaintance or friend who is an immigrant") with immigrants, attention paid to news on migrants, opinion on media approach on migrants, perception of own skill level.

The majority of socio-demographic questions will be asked at the end of the survey. However, to conduct some heterogeneous analysis, some of these variables will be collected before the treatment. In particular, we will test possible heterogeneous impacts with respect to perceived inequality, political orientation, self-reported skill level and own attitudes towards migrants (asking a question on beliefs about the impact of immigrants on Italy's welfare or own opinion about the number of immigrants to be allowed to enter in Italy). Given that one of the objectives of the study is to test if our treatments correct misperceived beliefs about natives' attitudes, we will also test possible heterogeneous im- 
pacts with respect to pre-treatment beliefs of natives' attitude. Some people may start with very negative baseline beliefs on attitudes of the ingroup. If our treatments have stronger effects on respondents' donation and petition signature for this sub-group of people, we can infer that the treatment was successfully able to change beliefs on peers' attitudes in the expected direction.

\section{Sample size and power}

We can determine the minimum effect size that we will be able to detect with statistical precision given our sample size. We take as reference for the power-calculation the data of a pilot conducted in the U.K. in August 2019. We calculate that a sample size of at least 1051 subjects per treatment would allow us to detect the previous 5 percentage point increase in donations with 95 percent confidence level and 80 percent power. This makes the overall sample of our analyses equal to around 5250 Italian respondents for the main survey. The survey firm in charge of collecting the data will select a representative sample of respondents with respect to pre-screened socio-demographic characteristics and will terminate the data collection once the target for the specific group is reached.

\section{Analysis}

The objective of the present study is two-fold. First, we aim at testing the effect of providing social information on incentivized pro-migration behaviour. Second, if we successfully influence behaviour, our second objective is to test the channel(s) through which the treatments positively influence behaviour. Our hypothesis is that the social information introduces an exogenous variation in subjects' beliefs on peers' (favourable) attitude towards migrants. Large peers' donation to a charity that strive in favour of migrant should deliver a clear sign that peers are not against migrants, and therefore exhibit a favourable attitude towards them. We will estimate the following equation:

$$
y_{i}=\beta_{0}+\sum_{a=1}^{A} \beta_{a} T_{i}^{n}+\beta_{2} Z_{i}+\varepsilon_{i}
$$

where $y_{i}$ : i) own donation; ii) intention to sign a petition; iii) effective signature of the petition. $T_{i}^{n}$ is a matrix of treatment variables. The treatment variable is equal to one if respondent $i$ receives Treatment $n$ and 0 otherwise. $Z_{i}$ is a matrix of individual characteristics, which include standard socio-demographic controls, a proxy for warmglow, altruism, and own's and peers' attitudes towards migrants.

In our analysis, we specifically measure the effect of the treatments on incentivized behaviours. Both donation and signature of a petition are costly choices that measure pro-migration behaviour. We could also test if the treatments affect the respondents' 
attitude toward migrants. However, given that this measure is not the result of a costly choice, our preferred outcome variables are the incentivized ones (i, ii, iii above).

Moreover, if we successfully influence behaviour, our second objective is to test the channel(s) through which the treatments positively influence behaviour. Our hypothesis is that the social information introduces an exogenous variation in subjects' beliefs on peers' (favourable) attitude towards migrants. Large peers' donation to a charity that strive in favour of migrant should deliver a clear sign that peers are not against migrants, and therefore exhibit a favourable attitude towards them.

To meet the second objective, $y_{i}$ is: iv) beliefs on the average donation level of other natives; v) beliefs on post-treatment peers' attitude. Moreover, as already described, we will conduct some heterogeneous analysis with respect to pre-treatment beliefs on natives' attitude to test a possible channel through which the treatments positively influence behaviour.

We will complement the reduced-form experimental evidence with structural estimates of the model parameters. The structural estimation allows us to decompose the share of giving that is due to a reduction in the perceived social distance between the ingroup and the outgroup - obtained through the information on peers' positive attitude - versus conformism. Given that our treatments intend to introduce an exogenous variation in beliefs about what others think about migrants (namely, beliefs on peers' attitudes), we need to identify whether subjects increase donations because they simply adhere to the social norm, or because they modify their perception of the ingroup-outgroup distance, and eventually quantify the weight of these two components in explaining the effectiveness of the treatment. 


\section{References}

Alesina, A., A. Miano, and S. Stantcheva (2018). Immigration and Redistribution. National Bureau of Economic Research, National Bureau of Economic Research.

Andreoni, J. (1990). Impure Altruism and Donations to Public Goods: A Theory of Warm-Glow Giving. The Economic Journal 100(401), 464-477.

Brader, T., N. A. Valentino, and E. Suhay (2008). What Triggers Public Opposition to Immigration? Anxiety, Group Cues, and Immigration Threat. American Journal of Political Science 52(4), 959-978.

Bursztyn, L., G. Egorov, and S. Fiorin (2017, May). From extreme to mainstream: How social norms unravel. Working Paper 23415, National Bureau of Economic Research.

Croson, R. T., F. Handy, and J. Shang (2010). Gendered giving: the influence of social norms on the donation behavior of men and women - Croson - 2010 - International Journal of Nonprofit and Voluntary Sector Marketing - Wiley Online Library. International Journal of Nonprofit and Voluntary Sector Marketing 15(2), 199-213.

Frey, B. S. and S. Meier (2004). Social Comparisons and Pro-Social Behavior: Testing "Conditional Cooperation" in a Field Experiment. The American Economic Review $94(5), 1717-1722$.

Hainmueller, J. and D. J. Hopkins (2014). Public Attitudes Toward Immigration. Annual Review of Political Science 17(1), 225-249.

Shang, J. and R. Croson (2009, October). A Field Experiment in Charitable Contribution: The Impact of Social Information on the Voluntary Provision of Public Goods. The Economic Journal 119(540), 1422-1439. 